\title{
Gestational Age Dependency of Essential Fatty Acids in Cord Plasma Cholesterol Esters and Triglycerides
}

\author{
EDDA B. HOVING, CHRISTIEN M. VAN BEUSEKOM, HENK J. NIJEBOER, AND \\ FRITS A. J. MUSKIET
}

Central Laboratory for Clinical Chemistry, University Hospital Groningen, 9700 RB Groningen. The Netherlands

\begin{abstract}
Plasma cholesterol ester and triglyceride fatty acid compositions of 38 singleton deliveries (23-42 wk), three twins (32, 39, and 40 wk), and their mothers were investigated. No gestational age-dependent changes occurred in maternal fatty acid compositions. Long-chain polyunsaturated fatty acids in fetal cholesterol esters and triglycerides were higher, and their precursors (18:2c, $\omega 6$ and 18:3c, $\omega 3$ ) were lower than those in corresponding maternal lipid classes. Except for 22:6c, $33,20: 2 c, \omega 6$, and 22:5c, $\omega 6$, all long-chain polyunsaturated fatty acids in fetal triglycerides increased with advancing gestation. Fetal triglyceride 22:6c, $\omega 3 / 22: 5 c, \omega 3$ ratio decreased, whereas 22:5c, $\omega 6 / 22: 4 c, \omega 6$ remained constant. Fetal cholesterol ester and triglyceride $20: 3 c, \omega 9$ contents were higher than those of corresponding maternal fractions and did not change with gestation. Triglyceride 18:2c, $\omega 6$ contents of babies with gestational ages of more than 34 wk were linearly related to those of their mothers. The data suggest that increasing triglyceride long-chain polyunsaturated fatty acid content with advancing gestation is partially caused by $\Delta 6$ - and $\Delta 5$-desaturase maturation in the liver. Constancy of $22: 6 c, \omega 3$ and $22: 5 c, \omega 6 / 22: 4 c, \omega 6$ and decrease of $22: 6 c, \omega 3 / 22: 5 c, \omega 3$ in triglycerides may point to low hepatic $\Delta 4$-desaturation. Transplacental transport of $20: 3 c, \omega 9$, followed by fetal conservation, should be considered. High 18:2c, $\omega 6$ and low 18:3c, $\omega 3$ intakes by the mother may unfavorably influence fetal production of 22:6c, $\omega 3$ in the liver. Because of low hepatic $\Delta 4$-desaturation capacity the influence may be small, however. (Pediatr Res 35: 461-469, 1994)
\end{abstract}

\section{Abbreviations}

CE, cholesterol ester

TG, triglyceride

FA, fatty acid

LCPUFA, long-chain polyunsaturated fatty acid

EFA, essential fatty acid

LCAT, lecithin:cholesterol acyltransferase

PC, phosphatidylcholine

AFP, $\alpha$-fetoprotein

The parent EFA, linoleic (18:2c, $\omega 6)$ and $\alpha$-linolenic $(18: 3 \mathrm{c}, \omega 3)$ acids, are partially converted into LCPUFA with 20 carbon atoms or more. LCPUFA biosynthetic routes comprise alterna-

Received December 9, 1992; accepted November 4, 1993.

Correspondence and reprint requests: Dr. F. A. J. Muskiet, Central Laboratory for Clinical Chemistry, University Hospital Groningen, P.O. Box 30001, 9700 RB Groningen. The Netherlands.

Supported in part by CC Friesland Frico Domo, Leeuwarden, The Netherlands. ting desaturation (by $\Delta 6-, \Delta 5-$, and $\Delta 4$-desaturation) and chain elongation (1). Recent data indicate that $\Delta 4$-desaturation of $22: 5 c, \omega 3$ is actually accomplished by initial chain elongation, followed by $\Delta 6$-desaturation and peroxisomal $\beta$-oxidation to yield 22:6c, $\omega 3$ (2). LCPUFA are structural components of cellular membranes and precursors of eicosanoids (prostaglandins, thromboxanes, and leukotrienes). They are essential for normal growth and development. The rapidly developing brain in the last trimester and 1st postnatal months (3-5) may especially be vulnerable to low LCPUFA status $(5,6)$.

To obtain EFA, the fetus is totally dependent on transport across the placenta. Daily requirements per kilogram of body weight are estimated at 400 and $50 \mathrm{mg} \mathrm{FA}$ of the $\omega 6-$ and $\omega 3$ series, respectively (7). There is little evidence that the fetus is able to fulfil LCPUFA demand, notably $22: 6 c, \omega 3$, by desaturation-chain elongation of parent EFA (8). The most convincing argument is that postnatal feeding with infant formulas that, in contrast to human milk, lack LCPUFA $(9-12)$ results in low LCPUFA levels in circulating lipids $(9,11,12)$, erythrocytes (911 ), and cerebral cortex phospholipids (6) compared with breastfed infants. Low neonatal LCPUFA status has been associated with decreased visual acuity (13), and low LCPUFA intake may be a factor in lower intelligence quotient of formula-fed premature infants compared with breast milk-fed counterparts (14).

Main sources for placental FA transport are maternal TG in VLDL (from the liver) and albumin-bound FFA (from adipose tissue). Placental uptake of FFA occurs by facilitated diffusion, after TG hydrolysis by lipoprotein lipase, or dissociation from albumin $(15,16)$. The origin of fetal plasma lipids is as yet uncertain. Under normal conditions the fetus is not considered to mobilize FA from adipose tissue $(16,17)$. Therefore, the identity of FA in fetal plasma lipids relates to the resultant of placental and hepatic FA: 1) uptake, 2) use for own structural requirements, 3) de novo synthesis, 4) oxidation, 5) modification (by desaturation, elongation, and chain shortening), 6) assembly to lipids, and 7) secretion into the fetal circulation. The role of the placenta in secretion of TG, phospholipids, and CE into fetal plasma is considered to be minor. Although it is known to produce apo B-100, cultured human trophoblasts did not release glycerolipids into the medium (15). Fetal plasma CE may therefore predominantly be synthesized by circulating LCAT (EC 2.3.1.43) that preferentially transfers FA from the $s n-2$ position of plasma PC to free cholesterol (18). FA supply to fetal liver may be restricted to FFA secreted by the placenta. Maintenance of plasma TG levels in term and premature infants during the 1st postnatal day (19) shows that at least during late gestation the liver is capable of VLDL secretion. Because of differences in half-lives of circulating CE (days) and TG (hours), fetal plasma CE FA composition may be expected to reflect long-term FA transfer across the placenta indirectly, whereas TG FA composition indirectly points at recent transfer.

The study of FA transport across the human placenta is 
difficult. FA measurements in maternal and fetal circulation (20-23) and fetal organs $(5,7)$ may provide insight, but they do not necessarily disclose the dynamics of the underlying processes. Gestational age-dependent FA contents of maternal and fetal plasma lipid classes with packed column gas chromatography have been reported (22). Taking advantage of the much higher separating power of capillary gas chromatography, we reexamined CE and TG FA compositions of cord and maternal plasma at delivery. Samples were collected from 38 singleton and three twin deliveries at 23-42 gestational wk. The data were evaluated in the light of existing knowledge on transplacental FA transport, EFA metabolism, early neonatal changes in circulating FA composition, and the presumed origin of cord plasma lipids.

\section{MATERIALS AND METHODS}

Subjects and samples. Cord and maternal blood samples from 503 spontaneous deliveries were obtained from a study on the incidence of hemoglobinopathies and thalassaemias on the Caribbean island of Curacao (Netherlands Antilles) (24). Samples from 38 singleton and three twin deliveries were selected (see below). Most of the population on Curacao is of West African descent. Dietary habits are essentially Western, with trigh intake of 18:2c,w6. Data on gender and birth weight were recorded. Gestational age was obtained from the time of last menstruation. The study conformed to local ethical standards and the Helsinki declaration of 1975 as revised in 1983.

Blood was collected in EDTA-containing Vacutainer tubes (Becton Dickinson Vacutainer Systems, Meylan, France) and centrifuged for $5 \mathrm{~min}$ at $1500 \times \mathrm{g}$. The plasma was immediately frozen at $-20^{\circ} \mathrm{C}$, transported to The Netherlands in dry ice, and stored at $-20^{\circ} \mathrm{C}$. All analyses were performed within $6 \mathrm{mo}$ and in one series. For FA analyses, only samples from babies with $\mathrm{HbAA}$ and $\mathrm{HbBarts} \leq 0.5 \%$ and mothers with HbAA [as determined by HPLC (24)] were considered. All samples from premature deliveries (gestational age $<38$ wk; $n=19$ ) and twin pregnancies $(n=3$; gestational ages 32,39 , and $40 \mathrm{wk})$ were analyzed. From the remaining term (38-42 wk) deliveries, a maximum of four mother-child couples per unit of gestational age (week) were chosen by random selection, giving a total of 19 term mother-singleton child couples. The whole study group was composed of 24 girls and 20 boys. Two infants, both born in 24 wk gestational age (birth weights 900 and $1300 \mathrm{~g}$ ), died after $5 \mathrm{~h}$ and $3 \mathrm{~d}$, respectively.

Methods. TG (without free glycerol blanking) and maternal total cholesterol levels in plasma were determined by enzymatic methods on an RA-1000 (Technicon Instruments Corporation, Tarrytown, NY). Fetal total and free cholesterol were determined by gas chromatography as previously described (25). Fetal plasma esterified cholesterol concentration and percentage free cholesterol were calculated.

Total plasma lipids were extracted from $150-\mu \mathrm{L}$ aliquots by the method of Folch et al. (26). CE and TG were isolated by solid-phase extraction (27). Transmethylation was conducted in methanol- $\mathrm{HCl}$ as previously described (28). Capillary gas chromatographic profiling of FA methyl esters (27) was performed on a $50 \mathrm{~m} \times 0.2 \mathrm{~mm}$ (inside diameter) apolar Ultra 1 crosslinked methyl silicone-coated column (Hewlett-Packard, Amstelveen, The Netherlands).

Data analysis and statistics. To allow comparison with data of Friedman et al. (22), we grouped the data from singleton deliveries into those from babies with gestational ages of 23-33 $\mathrm{wk}[n=7$, including four very premature deliveries $(<28 \mathrm{wk})]$, premature deliveries (35-37 wk; $n=12$ ), and term deliveries (38-42 wk; $n=19)$. Gestational age dependency was investigated with the Spearman rank correlation test. Correlations between FA percentages were studied by orthogonal regression analysis (29).

\section{RESULTS}

Study group and plasma lipids. Compared with the curves described by Lubchenco et al. (30), most of the babies investigated were found to be appropriate for gestational age (Fig. 1A). Data on gender, birth weight, and cord plasma lipids are given in Table 1. Fetal plasma free cholesterol and TG concentrations did not change with advancing gestation, whereas total and esterified cholesterol increased. Lower cholesterol esterification percentages were notable in babies with gestational ages of 2333 wk (Fig. 1B).

Maternal plasma total cholesterol levels were $4.39 \pm 1.32$ (23$33 \mathrm{wk} ; n=7), 5.52 \pm 0.73(35-37 \mathrm{wk} ; n=12)$, and $5.85 \pm 1.86$ $\mathrm{mmol} / \mathrm{L}$ (38-42 wk; $n=19)$. The changes with gestation were not significant (Spearman $p>0.05$ ). In the same period TG concentrations increased from $1.23 \pm 0.71$ to $1.76 \pm 0.35$ and $1.99 \pm 0.52 \mathrm{mmol} / \mathrm{L}$ (Spearman $p<0.05$ )

Plasma CE and TG FA compositions. Tables 2 and 3 show FA compositions of CE (Table 2) and TG (Table 3 ) isolated from cord plasma and maternal plasma. Data for maternal plasma did not show gestational age-dependent changes. They were therefore taken as a whole. In both cord plasma CE and TG there were gestational age-dependent increases of 18:2c, $\omega 6$ (Fig. $2 A$ and $E$ ), 18:3c, $\omega 3$ (Fig. $2 B$ and $F$ ), 20:4c, $\omega 6$ (Fig. $2 C$ and $G$ ), $20: 3 \mathrm{c}, \omega 6$, sum of $\omega 6-\mathrm{FA}$, LCPUFA- $\omega 6$, polyunsaturated FA, and LCPUFA, whereas 18:1c, $\omega 9$, sum- $\omega 9$ and monounsaturated FA decreased.

Except for $22: 6 c, \omega 3,20: 2 c, \omega 6$, and $22: 5 c, \omega 6$, gestational agedependent increases of all individual LCPUFA of the $\omega 3$ and $\omega 6$ series, as well as their parent EFA 18:3c, $\omega 3$ and 18:2c, $\omega 6$, were noted in cord plasma TG (Fig. 2, Table 3). Constancy of 22:6c, $\omega 3$ in cord plasma TG (Fig. $2 H$ ) was accompanied by a decrease in $22: 6 \mathrm{c}, \omega 3 / 22: 5 \mathrm{c}, \omega 3$ ratio (Fig. $3 A$ ) and no changes in the $22: 5 c, \omega 6 / 22: 4 c, \omega 6$ ratio (Fig. $3 B$ ). These ratios are parameters of $\Delta 4$-desaturation activity. They did not change in maternal plasma TG (Fig. $3 C$ and $D$, respectively).

Relationship between FA contents of maternal and cord plasma TG. Premature and term mother-child couples (35-42 wk) showed a linear relationship between 18:2c, $\omega 6$ contents of their plasma TG. This finding was not the case for mother-child couples with gestational ages of 23-33 wk (Fig. 4). A similar relationship between CE 18:2c, $\omega 6$ of term (38-42 wk) neonates and their mothers was noted. In the whole group of 38 motherchild couples a linear relationship existed between percentages $20: 5 c, \omega 3$ in maternal and neonatal CE. No other relationships were observed between maternal and cord FA contents in corresponding lipids.

Cord plasma CE and TG FA compositions of twins. Birth weights (in grams) of the three twins were 1260, 1420 (32 wk); 2450,2800 (39 wk); and 2450, 2540 (40 wk). Comparison of FA contents of each child couple showed remarkable differences for all EFA. Apart from CE 18:3c, $\omega 3$ content, the heaviest child was found to harbor the highest EFA contents in both cord plasma $\mathrm{CE}$ and TG (for selected data see Figs. 1-4). For the sum of LCPUFA of the $\omega 3$ - and $\omega 6$-series in the TG fraction these differences amounted to a factor 1.6-3.5. Relative to the parent EFA the heaviest child contained the highest TG LCPUFA percentages. Higher LCPUFA contents corresponded with lower monounsaturated FA, notably $18: 1 \mathrm{c}, \omega 9$, in fetal TG.

\section{DISCUSSION}

We investigated cord and maternal plasma CE and TG FA compositions of 38 singleton $(23-42 \mathrm{wk})$ and three twin (32-40 wk) deliveries. Samples were selected from 503 spontaneous deliveries on the Caribbean island of Curacao (Netherlands Antilles). Special emphasis was given to the course of CE and TG LCPUFA contents with advancing gestation. Cord plasma CE predominantly derive from plasma LCAT activity, whereas its TG may predominantly derive from the liver. We did not addi- 

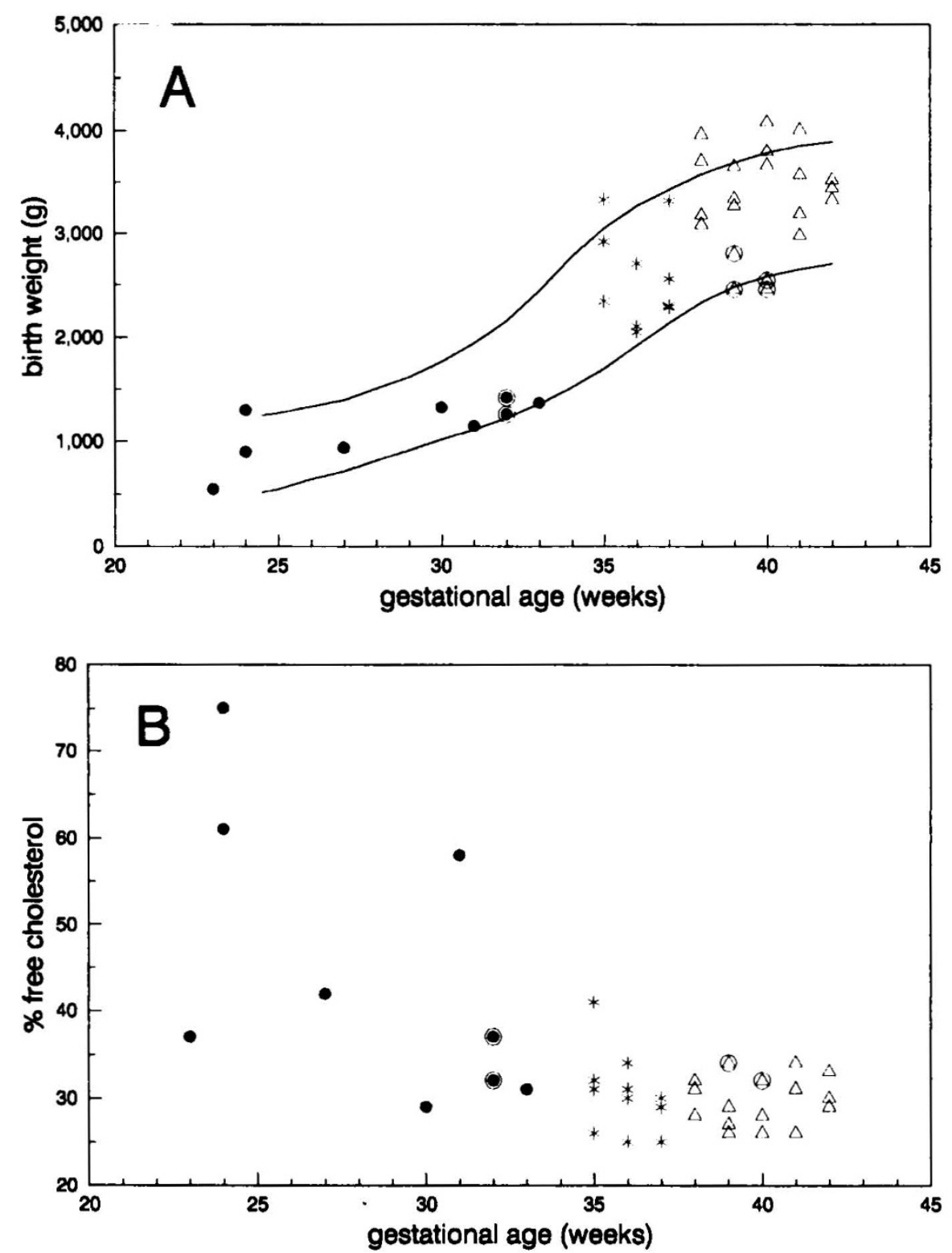

Fig. 1. Birth weight $(A)$ and percentage free cholesterol in cord plasma $(B)$ as a function of gestational age. The lines in $A$ represent the 10th and 90 th percentiles according to Lubchenco et al. (30). $\cdot$, gestational age $23-33 \mathrm{wk}$; , gestational age 35-37 wk; $\Delta$, gestational age 38-42 wk; encircled symbols indicate data from three twins.

Table 1. Data on babies and their cord plasma lipids*

\begin{tabular}{|c|c|c|c|c|c|c|c|}
\hline & \multicolumn{2}{|c|}{$23-33 \mathrm{wk}(n=7)$} & \multicolumn{2}{|c|}{$35-37 \mathrm{wk}(n=12)$} & \multicolumn{2}{|c|}{$38-42$ wk $(n=19)$} & \multirow{2}{*}{$\begin{array}{l}\text { Spearman } \\
p<0.05 \\
\end{array}$} \\
\hline & Mean & $\mathrm{SD}$ & Mean & SD & Mean & SD & \\
\hline Gestational age (wk) & 27.4 & 4.0 & 36.0 & 0.9 & 40.0 & 1.5 & + \\
\hline Gender (male/female) & $5 / 2$ & & $5 / 7$ & & $9 / 10$ & & \\
\hline Birth weight (g) & 1076 & 297 & 2590 & 469 & 3319 & 887 & + \\
\hline TG $(\mathrm{mmol} / \mathrm{L})$ & 0.46 & 0.12 & 0.44 & 0.15 & 0.58 & 0.29 & \\
\hline Total cholesterol $(\mathrm{mmol} / \mathrm{L})$ & 1.26 & 0.48 & 1.75 & 0.50 & 1.86 & 0.58 & + \\
\hline Free cholesterol $(\mathrm{mmol} / \mathrm{L})$ & 0.58 & 0.32 & 0.52 & 0.11 & 0.55 & 0.19 & \\
\hline Esterified cholesterol $(\mathrm{mmol} / \mathrm{L})$ & 0.69 & 0.40 & 1.23 & 0.41 & 1.32 & 0.40 & + \\
\hline Free cholesterol (\%) & 47.6 & 17.3 & 30.4 & 4.6 & 29.4 & 2.6 & - \\
\hline
\end{tabular}

* Data are grouped according to babies with gestational ages of 23-33 wk, 35-37 wk, and term (38-42 wk) deliveries. Data for the three twins are not included. Gestational age dependency was investigated with the Spearman rank correlation test. + , positive relationship; - , negative relationship.

tionally analyze plasma total phospholipid FA composition. Plasma phospholipids are mainly composed of PC. Therefore, plasma CE FA composition indirectly relates to plasma PC $s n-2$ FA composition, with LCAT transfer preferences (18) as "weight factors."

Study Group and Plasma Lipids. As previously established
(15, 31), maternal TG levels were found to increase during gestation, whereas the usually encountered moderate increase of maternal cholesterol did not reach significance. The course of fetal TG, but not esterified cholesterol, is consistent with data from Friedman et al. (22). The higher percentage free cholesterol in most babies with gestational ages of 23-33 wk (Fig. $1 B$, Table 
Table 2. Fatty acid compositions (in mol\%) of cholesterol esters in cord and maternal plasma*

\begin{tabular}{|c|c|c|c|c|c|c|c|c|c|}
\hline \multirow[b]{3}{*}{ FA } & \multicolumn{7}{|c|}{ Newborns } & \multirow{2}{*}{\multicolumn{2}{|c|}{$\begin{array}{l}\text { Mothers: } \\
23-42 \text { wk } \\
(n=38)\end{array}$}} \\
\hline & \multicolumn{2}{|c|}{$\begin{array}{c}23-33 \mathrm{wk} \\
(n=7)\end{array}$} & \multicolumn{2}{|c|}{$\begin{array}{c}35-37 \mathrm{wk} \\
(n=12)\end{array}$} & \multicolumn{2}{|c|}{$\begin{array}{c}38-42 \text { wk } \\
(n=19)\end{array}$} & \multirow{2}{*}{$\begin{array}{c}\text { Spearman } \\
p<0.05\end{array}$} & & \\
\hline & Mean & SD & Mean & SD & Mean & SD & & Mean & SD \\
\hline $14: 0$ & 1.24 & 0.30 & 0.98 & 0.12 & 1.09 & 0.14 & & 0.89 & 0.28 \\
\hline $16: 0$ & 24.65 & 3.36 & 21.84 & 1.64 & 21.76 & 1.96 & & 15.46 & 1.03 \\
\hline 18:0 & 4.35 & 1.15 & 3.31 & 0.71 & 3.08 & 0.73 & - & 1.05 & 0.48 \\
\hline $18: 3 c, \omega 3$ & 0.17 & 0.11 & 0.23 & 0.09 & 0.28 & 0.15 & + & 0.60 & 0.18 \\
\hline $20: 5 c, \omega 3$ & 0.17 & 0.07 & 0.16 & 0.05 & 0.18 & 0.05 & & 0.22 & 0.09 \\
\hline $22: 6 c, \omega 3$ & 0.52 & 0.22 & 0.74 & 0.17 & 1.08 & 0.31 & + & 0.45 & 0.14 \\
\hline Sum-w3 & 0.86 & 0.25 & 1.12 & 0.25 & 1.54 & 0.36 & + & 1.26 & 0.29 \\
\hline LCPUFA- $\omega 3$ & 0.69 & 0.24 & 0.90 & 0.20 & 1.26 & 0.33 & + & 0.67 & 0.20 \\
\hline $18: 2 c, \omega 6$ & 11.05 & 2.46 & 15.85 & 2.32 & 18.23 & 2.61 & + & 52.29 & 4.41 \\
\hline $18: 3 c, \omega 6$ & 0.55 & 0.24 & 0.65 & 0.19 & 0.71 & 0.20 & & 0.33 & 0.17 \\
\hline $20: 3 c, \omega 6$ & 0.65 & 0.22 & 1.06 & 0.18 & 1.34 & 0.27 & + & 0.66 & 0.14 \\
\hline $20: 4 c, \omega 6$ & 8.42 & 2.10 & 11.17 & 2.29 & 12.75 & 2.40 & + & 5.63 & 1.28 \\
\hline $22: 5 c, \omega 6$ & 0.11 & 0.05 & 0.13 & 0.03 & 0.16 & 0.04 & + & 0.04 & 0.02 \\
\hline Sum-w6 & 20.77 & 4.31 & 28.87 & 4.01 & 33.18 & 4.94 & + & 58.94 & 3.85 \\
\hline LCPUFA- $\omega 6$ & 9.17 & 2.25 & 12.36 & 2.36 & 14.24 & 2.60 & + & 6.33 & 1.35 \\
\hline $16: 1 c, \omega 7$ & 8.89 & 2.06 & 8.99 & 2.29 & 8.23 & 2.07 & & 3.42 & 1.55 \\
\hline $18: 1 c, \omega 7$ & 4.08 & 0.41 & 4.26 & 0.41 & 3.84 & 0.41 & & 1.51 & 0.24 \\
\hline Sum- $\omega 7$ & 12.97 & 2.00 & 13.26 & 2.56 & 12.07 & 2.30 & & 4.93 & 1.58 \\
\hline $18: 1 c, \omega 9$ & 34.85 & 6.01 & 30.26 & 2.53 & 26.92 & 2.85 & - & 17.41 & 2.20 \\
\hline $20: 3 c, \omega 9$ & 0.31 & 0.16 & 0.37 & 0.12 & 0.35 & 0.14 & & 0.06 & 0.03 \\
\hline Sum- $\omega 9$ & 35.16 & 5.94 & 30.62 & 2.61 & 27.27 & 2.93 & - & 17.47 & 2.22 \\
\hline SAFA & 30.24 & 4.59 & 26.13 & 2.06 & 25.93 & 2.22 & - & 17.40 & 1.33 \\
\hline MUFA & 47.82 & 6.75 & 43.51 & 4.34 & 39.00 & 4.46 & - & 22.34 & 3.40 \\
\hline PUFA & 21.94 & 4.57 & 30.36 & 4.07 & 35.07 & 5.12 & + & 60.26 & 3.74 \\
\hline LCPUFA & 9.86 & 2.45 & 13.26 & 2.47 & 15.51 & 2.88 & + & 6.99 & 1.51 \\
\hline $20: 3 c, \omega 9 / 20: 4 c, \omega 6$ & 0.04 & 0.01 & 0.04 & 0.02 & 0.03 & 0.02 & & 0.01 & 0.00 \\
\hline
\end{tabular}

* Data for the three twins are not included. Because there was no gestational age dependency, data for maternal plasma were grouped. Sum- $\omega x$, sum of indicated series of fatty acids; LCPUFA- $\omega$ X, sum of long-chain polyunsaturated fatty acids with 20 carbon atoms or more of indicated series of fatty acids; SAFA, saturated fatty acids; MUFA, monounsaturated fatty acids; PUFA, polyunsaturated fatty acids; LCPUFA, sum of long-chain polyunsaturated fatty acids of the $\omega 3$ - and $\omega 6$-series with 20 carbon atoms or more. Gestational age dependency was investigated with the Spearman rank correlation test. + , positive relationship; - , negative relationship.

1) was previously found (32) and is caused by lower LCAT activity $(32,33)$. As in adults, circulating LCAT in newborns most likely derives from the liver, which implies immaturity of this liver function parameter in babies with gestational ages of 23-33 wk. LCAT deficiency and severe hepatic dysfunction in adults cause accumulation of free cholesterol in erythrocytes and target cell formation (34). It is caused by an increase of plasma free cholesterol/phospholipid ratio. Because in the fetus neither free cholesterol concentrations (Table 1) nor phospholipid levels (22) change, no such effect may be expected in the fetus.

Plasma CE and TG FA Compositions. LCPUFA. Percentages of LCPUFA of the $\omega 3$ - and $\omega 6$-series in cord plasma CE and TG are higher and those of their parent precursors $(18: 3 \mathrm{c}, \omega 3$ and $18: 2 c, \omega 6)$ are lower compared with corresponding maternal fractions (Tables 2 and 3). Higher LCPUFA percentages in the fetal circulation have previously been described (20-23), and the underlying process has been named biomagnification (35). Both LCPUFA and their precursor percentages increase with advancing gestation, notably at the expense of monounsaturated FA and saturated FA (CE only).

In the light of the presumed placental-hepatic origin of fetal plasma TG FA, the data suggest preferential transfer of LCPUFA compared with their parent precursors. An explanation by high desaturation-chain elongation activity in placenta is unlikely. At 18-22 wk of gestation placental microsomes lack $\Delta 5$ - and $\Delta 6$ desaturase activities $(9,15)$. High LCPUFA synthetic activity in the liver is also unlikely. Postnatal feeding with formulas that lack LCPUFA but are high in parent precursors leads to plasma lipid and erythrocyte LCPUFA contents that are below those encountered in infants fed LCPUFA-containing human milk (9, 11). It is possible, however, that at least part of the increase of TG LCPUFA reflects development of $\Delta 5$ - and $\Delta 6$-desaturase activities in the liver (see below).

The mechanism for preferential LCPUFA transfer is as yet unknown, although it has been suggested that it involves AFP (36). AFP exhibits high affinity for LCPUFA (37). The present notion is that placental FA uptake takes place by facilitated diffusion of maternal albumin-bound FFA and those released from VLDL-TG by placental lipoprotein lipase $(15,16)$. Studies in monkeys showed that FFA are both transferred from mother to fetus and from fetus to mother (38). No experiments regarding placental perfusion have been performed with LCPUFA in maternal circulation and fat-free AFP in fetal circulation. Trapping of LCPUFA by AFP in the fetal circulation, followed by receptormediated uptake (36) in growing tissues, may offer an explanation for the underlying mechanism of biomagnification and subsequent LCPUFA deposition in specific organs, respectively.

Levels of 22:6c, $\omega 3$ and $\Delta 4$-desaturation. The only fetal TG LCPUFA percentages that did not increase with advancing gestation were those of $22: 6 \mathrm{c}, \omega 3$ (Fig. $2 H$ ), 20:2c, $\omega 6$, and 22:5c, $\omega 6$ (Table 3). Because maternal TG and CE FA compositions did not change with gestation, it cannot be explained by changing 
Table 3. Fatty acid compositions (in mol\%) of TG in cord and maternal plasma*

\begin{tabular}{|c|c|c|c|c|c|c|c|c|c|}
\hline \multirow[b]{3}{*}{$\mathrm{FA}$} & \multicolumn{7}{|c|}{ Newborns } & \multirow{2}{*}{\multicolumn{2}{|c|}{$\begin{array}{l}\text { Mothers: } \\
23-42 \mathrm{wk} \\
(n=38)\end{array}$}} \\
\hline & \multicolumn{2}{|c|}{$\begin{array}{c}23-33 \text { wk } \\
(n=7)\end{array}$} & \multicolumn{2}{|c|}{$\begin{array}{c}35-37 \mathrm{wk} \\
(n=12)\end{array}$} & \multicolumn{2}{|c|}{$\begin{array}{c}38-42 \text { wk } \\
(n=19)\end{array}$} & \multirow{2}{*}{$\begin{array}{c}\text { Spearman } \\
p<0.05\end{array}$} & & \\
\hline & Mean & SD & Mean & SD & Mean & SD & & Mean & SD \\
\hline 14:0 & 1.83 & 0.61 & 2.13 & 0.64 & 2.22 & 0.52 & & 2.04 & 0.92 \\
\hline $16: 0$ & 30.15 & 5.24 & 29.83 & 2.67 & 30.84 & 1.98 & & 34.16 & 2.28 \\
\hline 18:0 & 6.12 & 2.71 & 5.19 & 1.41 & 4.96 & 0.93 & & 4.15 & 0.83 \\
\hline $20: 0$ & 0.16 & 0.05 & 0.15 & 0.08 & 0.14 & 0.08 & & 0.11 & 0.05 \\
\hline $18: 3 c, \omega 3$ & 0.16 & 0.07 & 0.41 & 0.16 & 0.45 & 0.13 & + & 0.71 & 0.19 \\
\hline $20: 5 c, \omega 3$ & 0.05 & 0.05 & 0.10 & 0.05 & 0.11 & 0.04 & + & 0.06 & 0.03 \\
\hline $22: 5 c, \omega 3$ & 0.08 & 0.05 & 0.18 & 0.08 & 0.23 & 0.07 & + & 0.09 & 0.04 \\
\hline $22: 6 c, \omega 3$ & 1.15 & 0.66 & 1.90 & 0.89 & 1.60 & 0.62 & & 0.44 & 0.17 \\
\hline Sum- $\omega 3$ & 1.44 & 0.71 & 2.59 & 0.97 & 2.39 & 0.72 & & 1.29 & 0.32 \\
\hline LCPUFA- $\omega 3$ & 1.28 & 0.73 & 2.18 & 0.96 & 1.94 & 0.68 & & 0.58 & 0.22 \\
\hline $18: 2 c, \omega 6$ & 6.68 & 1.42 & 11.99 & 3.48 & 12.41 & 2.60 & + & 19.07 & 3.31 \\
\hline $20: 2 c, \omega 6$ & 0.17 & 0.10 & 0.28 & 0.08 & 0.26 & 0.06 & & 0.30 & 0.08 \\
\hline $18: 3 c, \omega 6$ & 0.20 & 0.08 & 0.39 & 0.15 & 0.45 & 0.19 & + & 0.10 & 0.04 \\
\hline $20: 3 c, \omega 6$ & 0.37 & 0.11 & 0.70 & 0.14 & 0.74 & 0.18 & + & 0.34 & 0.14 \\
\hline $20: 4 c, \omega 6$ & 1.67 & 0.63 & 2.89 & 0.85 & 3.36 & 0.98 & + & 1.13 & 0.46 \\
\hline $22: 4 c, \omega 6$ & 0.34 & 0.17 & 0.50 & 0.11 & 0.61 & 0.15 & + & 0.12 & 0.04 \\
\hline $22: 5 c, \omega 6$ & 0.63 & 0.36 & 1.03 & 0.27 & 0.97 & 0.27 & & 0.12 & 0.05 \\
\hline Sum-w6 & 10.05 & 2.18 & 17.77 & 4.12 & 18.79 & 3.71 & + & 21.17 & 3.36 \\
\hline LCPUFA- $\omega 6$ & 3.17 & 1.16 & 5.40 & 1.04 & 5.93 & 1.48 & + & 2.00 & 0.64 \\
\hline $16: 1 c, \omega 7$ & 6.03 & 1.35 & 5.93 & 1.45 & 6.26 & 1.00 & & 2.99 & 0.96 \\
\hline $18: 1 c, \omega 7$ & 5.34 & 1.01 & 4.57 & 0.60 & 4.19 & 0.90 & - & 3.38 & 0.52 \\
\hline Sum- $\omega 7$ & 11.37 & 2.32 & 10.50 & 1.84 & 10.45 & 1.49 & & 6.37 & 1.29 \\
\hline $18: 1 c, \omega 9$ & 37.64 & 5.75 & 30.71 & 1.79 & 29.22 & 2.91 & - & 30.21 & 2.63 \\
\hline $20: 1 c, \omega 9$ & 0.50 & 0.11 & 0.42 & 0.17 & 0.30 & 0.12 & - & 0.36 & 0.09 \\
\hline $20: 3 c, \omega 9$ & 0.75 & 0.33 & 0.70 & 0.22 & 0.70 & 0.25 & & 0.13 & 0.05 \\
\hline Sum- $\omega 9$ & 38.88 & 5.93 & 31.83 & 1.98 & 30.22 & 3.04 & - & 30.71 & 2.67 \\
\hline SAFA & 38.26 & 8.11 & 37.31 & 3.59 & 38.15 & 2.94 & & 40.46 & 3.07 \\
\hline MUFA & 49.51 & 7.98 & 41.63 & 2.91 & 39.97 & 4.10 & - & 36.94 & 3.01 \\
\hline PUFA & 12.23 & 2.82 & 21.06 & 4.39 & 21.88 & 4.20 & + & 22.60 & 3.46 \\
\hline LCPUFA & 4.46 & 1.85 & 7.58 & 1.60 & 7.88 & 1.93 & + & 2.58 & 0.85 \\
\hline $20: 3 c, \omega 9 / 20: 4 c, \omega 6$ & 0.47 & 0.19 & 0.26 & 0.11 & 0.22 & 0.12 & - & 0.12 & 0.04 \\
\hline $22: 6 c, \omega 3 / 22: 5 c, \omega 3$ & 15.33 & 6.96 & 11.76 & 4.69 & 7.32 & 2.52 & - & 5.31 & 1.99 \\
\hline $22: 5 c, \omega 6 / 22: 4 c, \omega 6$ & 2.01 & 1.02 & 2.13 & 0.57 & 1.62 & 0.30 & & 1.02 & 0.26 \\
\hline
\end{tabular}

* Data for the three twins are not included. The $22: 6 c, \omega 3 / 22: 5 c, \omega 3$ and $22: 5 c, \omega 6 / 22: 4 c, \omega 6$ are parameters for $\Delta 4$-desaturation activity. For other abbreviations and statistics, see legend of Table 2.

placental supply. Constancy of fetal TG 22:6c, $\omega 3$ and 22:5c, $\omega 6$ contrasts with encountered increases of CE 22:6c, $\omega 3$ (Fig. 2D) and 22:5c, $\omega 6$ (Table 2). Gestational age-dependent increase of plasma phospholipid $22: 6 c, \omega 3$ content (22) may explain its concomitant increase in circulating CE via LCAT activity. The data suggest that with advancing gestation $22: 6 c, \omega 3$ and $22: 5 c, \omega 6$ are increasingly transported via phospholipids or directly from placenta to developing organs as FFA, but it does not seem a plausible option.

An alternative and more likely explanation for deviant behavior of fetal TG 22:6c, $\omega 3$ and 22:5c, $\omega 6$ with advancing gestation is retarding $\Delta 4$-desaturation development in fetal liver compared with $\Delta 5$ - and $\Delta 6$-desaturase developments. Despite increasing hepatic supply of parent EFA and increase of their desaturationchain elongation products up to $22: 5 c, \omega 3$ and $22: 4 c, \omega 6$ in fetal TG, the 22:6c, $\omega 3 / 22: 5 c, \omega 3$ ratio decreased from $15.33(23-33$ wk) to 7.32 (38-42 wk) (Table 3). No increase of 22:5c,w6/ $22: 4 c, \omega 6$ ratio (caused by induction of $\Delta 4$-desaturation activity) was noted, as seen in 22:6c, $\omega 3$ deficiency caused by $\omega 3$-shortage (9). These observations are consistent with postnatal comparative studies of human milk and infant formulas. From a percentage point of view, postnatal feeding with infant formulas that lack LCPUFA leads to a notable drop of $22: 6 c, \omega 3$ in erythrocytes (9$11)$, plasma lipids $(9,11,12)$, and brain $(6)$, whereas brain $20: 4 c, \omega 6$ levels were somewhat higher (6). Except for one study (13), none of the studies in which infant formula 18:2c, $\omega 6$ / $18: 3 \mathrm{c}, \omega 3$ ratio was varied caused plasma and erythrocyte $22: 6 c, \omega 3$ levels to rise to those of breast-fed infants (11). With decreasing formula $18: 2 c, \omega 6 / 18: 3 c, \omega 3$ ratio, plasma and erythrocyte 20:5c, $\omega 3$ and $22: 5 c, \omega 3$ levels did, however, increase. At an 18:2c, $\omega 6 / 18: 3 c, \omega 3$ ratio of approximately $3,20: 5 c, \omega 3$ and $22: 5 c, \omega 3$ levels even exceeded those encountered in breast-fed infants (11). Consequently, chain elongation-desaturation from $18: 3 c, \omega 3$ up to $22: 5 c, \omega 3$ in newborns seems possible. It implies $\Delta 6$ - and $\Delta 5$-desaturase activity, presumably in the liver. The gestational age-dependent gradual increase of EFA of the $\omega 6$ series up to $22: 4 c, \omega 6$ and the $\omega 3$-series up to $22: 5 c, \omega 3$ in fetal TG may, therefore, partially be caused by maturation of $\Delta 6$ - and $\Delta 5$-desaturases in the liver. Because $\Delta 4$-desaturation involves chain elongation, $\Delta 6$-desaturation, and peroxisomal $\beta$-oxidation (2), retarding development of the latter may be the causal factor of deviant TG $22: 6 c, \omega 3$ and $22: 5 c, \omega 6$ behaviors. Insufficient 
cholesterol esters
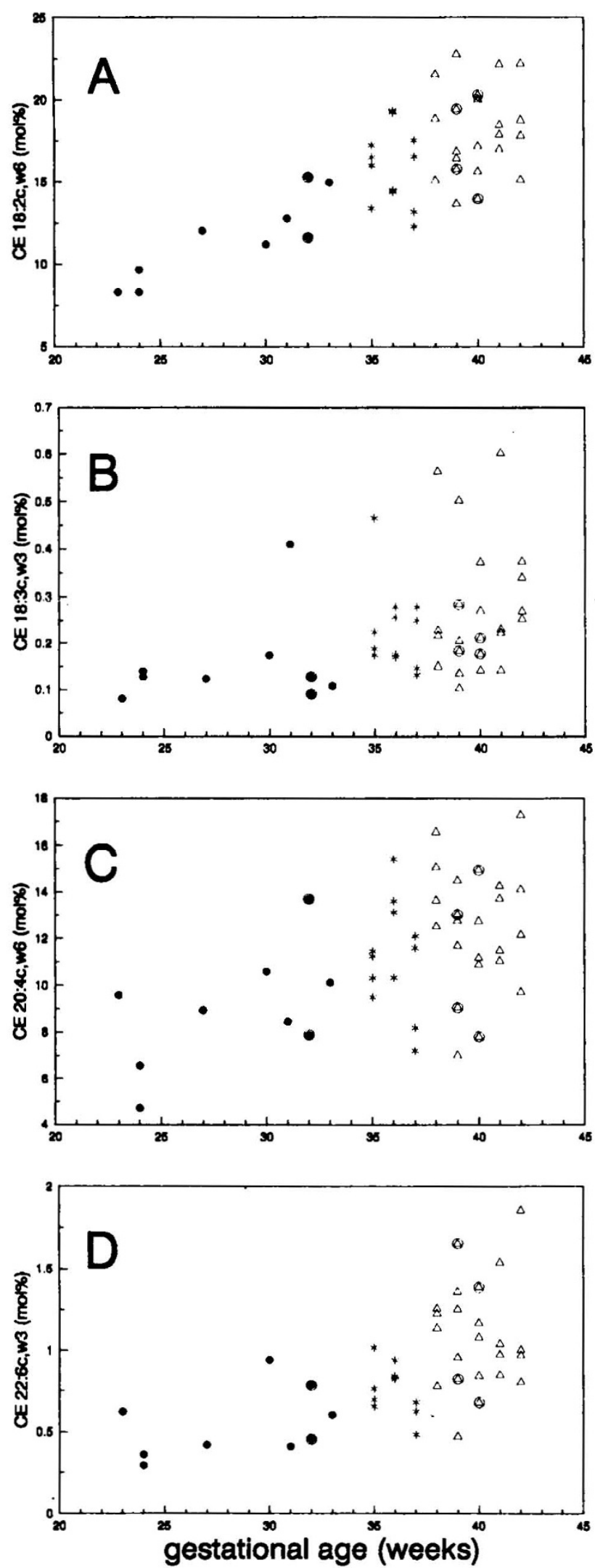

triglycerides
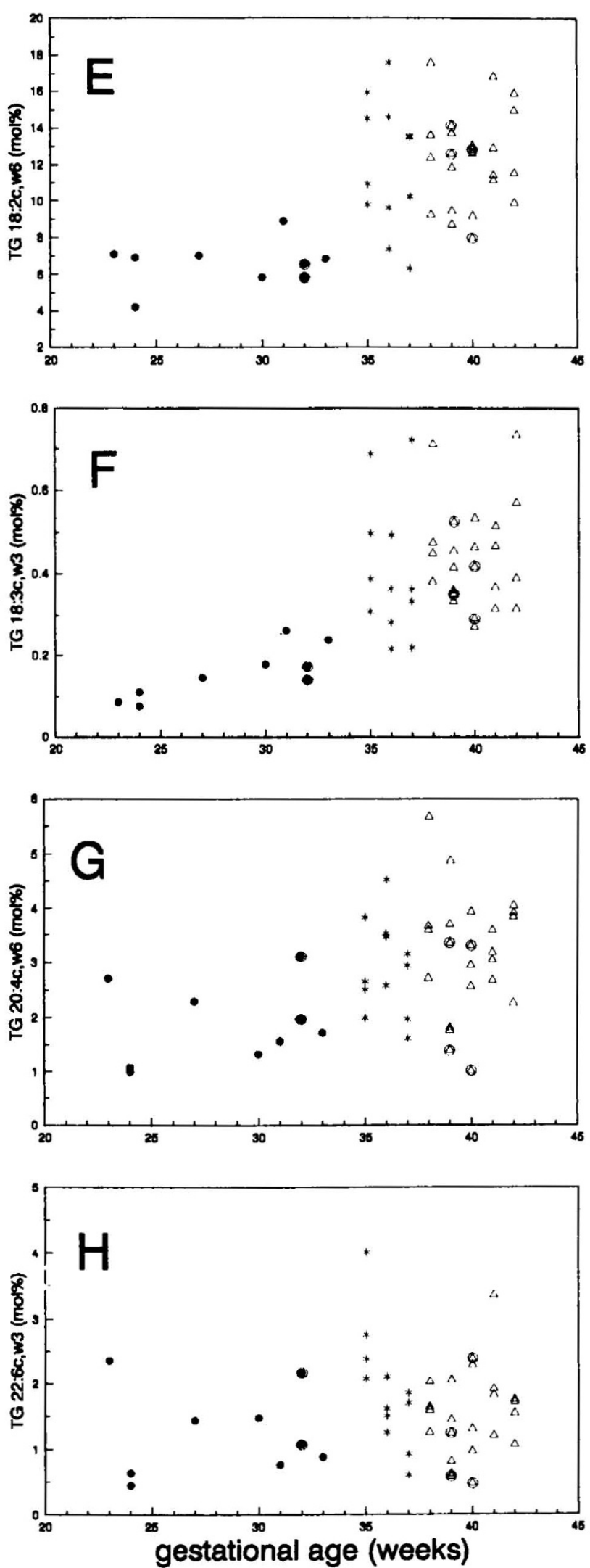

Fig. 2. Essential fatty acids (in mol\%) in cord plasma CE (left panels) and TG (right panels) as a function of gestational age. Except for 22:6c, 33 in the TG fraction $(H)$, all essential FA increased with gestational age (Spearman $p<0.05$, Tables 2 and 3). For symbols, see legend of Figure 1. 
cord plasma
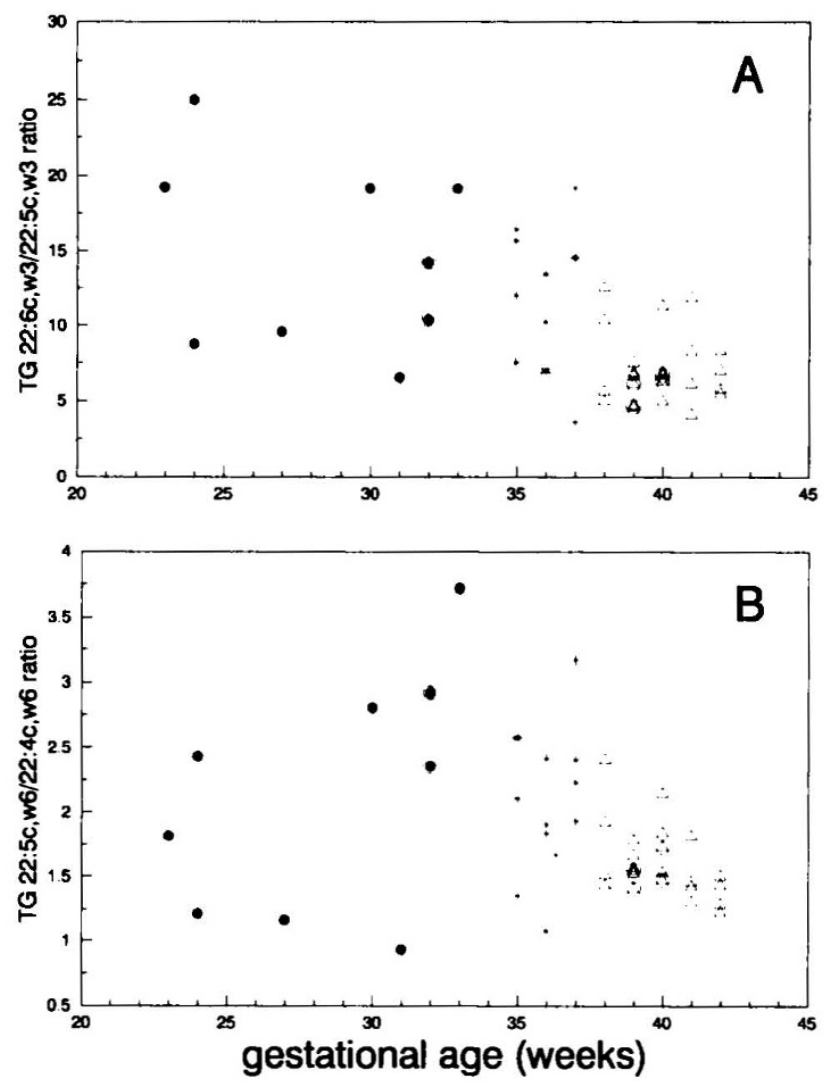

maternal plasma
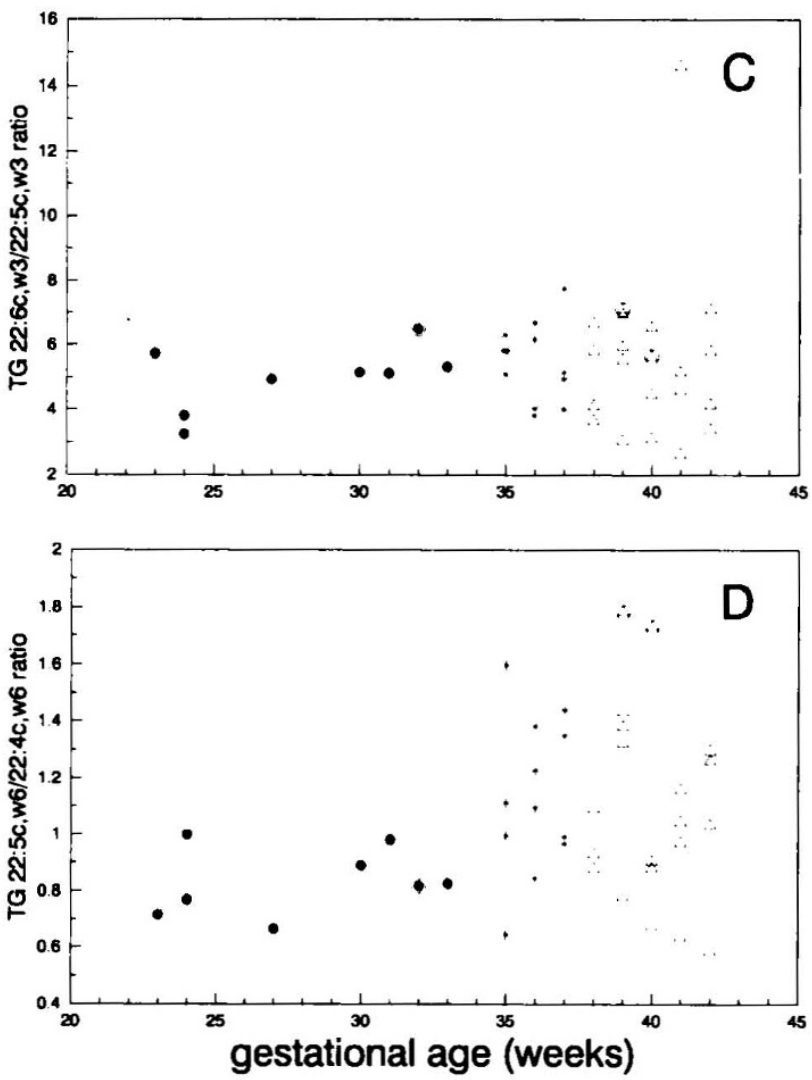

Fig. 3. Parameters of $\Delta 4$-desaturation activity as derived from cord (left panels) and maternal (right panels) plasma TG as a function of gestational age. Data represent molar ratios. The 22:6c, $\omega 3 / 22: 5 \mathrm{c} \omega 3$ ratio in cord plasma decreased with advancing gestation (Spearman $p<0.05$; Table 3). No changes occurred in the $22: 5 c, \omega 6 / 22: 4 c, \omega 6$ ratios in cord and maternal plasma and $22: 6 c, \omega 3 / 22: 5 c \omega 3$ ratio in maternal plasma. For symbols, see legend of Figure 1.

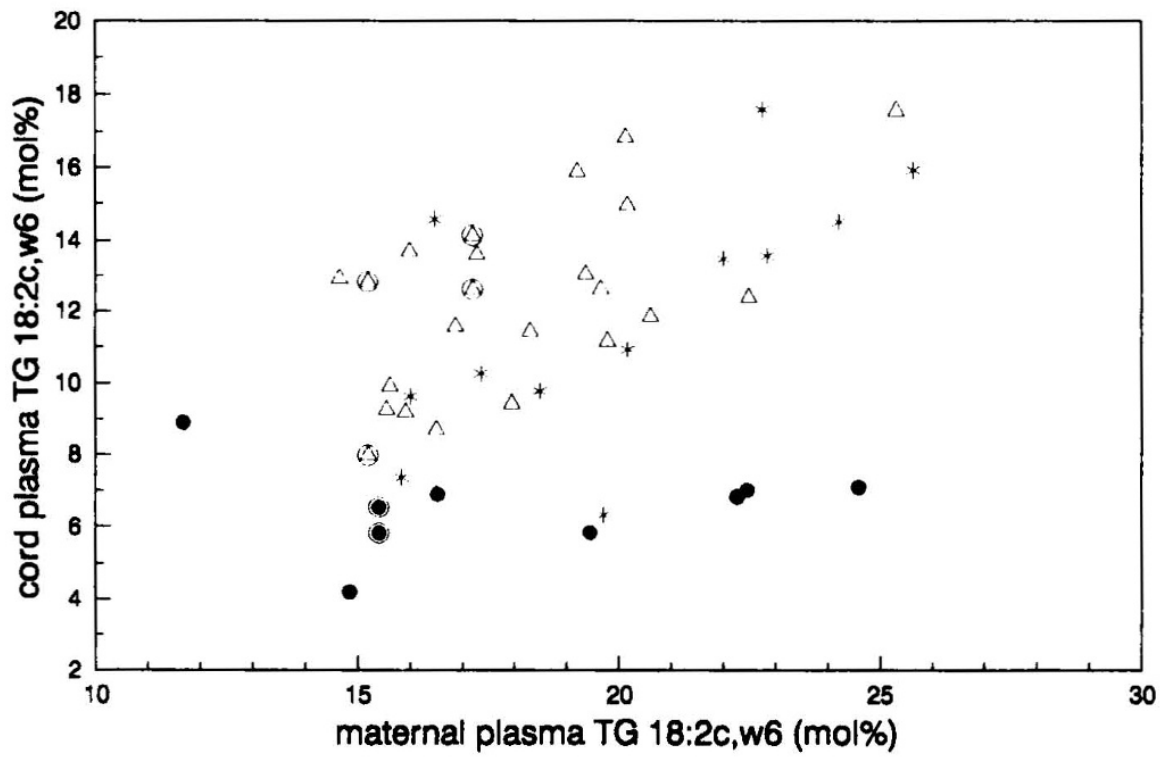

Fig. 4. Relationship between linoleic acid contents (in mol\%) of cord and maternal plasma TG. Cord plasma TG 18:2c, $\omega 6$ contents of premature $(*, 35-37 \mathrm{wk})$ and term $(\Delta, 38-42 \mathrm{wk})$ newborns were linearly related to those of maternal plasma (orthogonal regression analysis: $\mathrm{y}=0.95 \times-5.85$, syx $=1.94$, sxy $=1.94, r=0.589, p<0.001)$. For other symbols, see legend of Figure 1 .

hepatic $\Delta 4$-desaturation activity may be the principal underlying reason to add $22: 6 \mathrm{c}, \omega 3$ to infant formula.

Levels of 20:3c, $\omega 9$. Sizeable 20:3c, $\omega 9$ percentages were observed in both fetal plasma CE (about $0.35 \mathrm{~mol} \%$; Table 2) and TG (about $0.71 \mathrm{~mol} \%$; Table 3 ). The levels did not change with gestation and were about 5 times higher than those in corresponding maternal lipids and those previously established in apparently healthy adults (27). Early neonatal erythrocyte 20:3c, $\omega 9$ levels are also higher (about 3-5 times) than those of the mother (23).

High 20:3c, $\omega 9$ levels in tissues and plasma lipids are associated 
with EFA deficiency (39). Umbilical arterial walls contain higher levels of $20: 3 c, \omega 9$ and lower levels of $\omega 6$-FA than umbilical venous walls, which has led to the suggestion of marginal downstream EFA status (40). The question arises as to what the origin of 20:3c, $\omega 9$ in fetal TG might be. Theoretically, it may derive from hepatic synthesis or hepatic uptake after transport across the placenta, with subsequent incorporation into TG and phospholipids. The increase of 20:4c, $\omega 6$ and decrease of 20:3c, $\omega 9 /$ $20: 4 c, \omega 6$ ratio in fetal plasma TG with advancing gestation (Table 3) suggest that the simultaneously increasing 18:2c, $\omega 6$ supply to the liver increasingly prevents conversion of $18: 1 c, \omega 9$ to $20: 3 c, \omega 9$ in favor of $20: 4 c, \omega 6$ production. However, prenatal transport of $20: 3 c, \omega 9$ across the placenta (e.g. via AFP trapping) and conservation in a period characterized by high LCPUFA demand and low FA oxidation may be an equally plausible explanation. Whatever the origin, incorporation of 20:3c, $\omega 9$ into membrane phospholipids is known to affect functional systems. Its incorporation into platelets, for example, is known to cause platelet hyperactivity (41).

Relationship between Linoleic Acid Contents of Maternal and Cord Plasma TG. The relationship between fetal and maternal TG 18:2c,w6 at gestational ages of 35-42 wk (Fig. 4) suggests that from the time of rapid transplacental FA transfer the resulting fetal plasma TG 18:2c, $\omega 6$ content is largely dependent on maternal plasma TG and FFA 18:2c, $\omega 6$ contents. It indicates that maternal dietary 18:2c, $\omega 6$ intake influences fetal 18:2c, $\omega 6$ content, as previously found in rabbits (15). FA of the $\omega 6$ and $\omega 3$ series compete for $\Delta 6$ - and $\Delta 5$-desaturases. Therefore, high intake of $18: 2 c, \omega 6$ and low intakes of $18: 3 c, \omega 3$ and other precursors of 22:6c, $\omega 3$ (e.g. fish), as in most Western countries, may be expected to influence the already limited fetal capacity to produce 22:6c, $\omega 3$ in an unfavorable manner. Because of the presumed low hepatic $\Delta 4$-desaturation activity, the influence on hepatic 22:6c, $\omega 3$ synthesis may be small, however.

Cord Plasma CE and TG FA Compositions of Twins. Data from the three twins suggest that, despite placental exposure to a given maternal plasma lipid FA composition, the resulting FA compositions of plasma CE and TG in twins may be considerably different. Higher EFA contents of the heaviest child may be related to better placental function, e.g. higher placental blood flow, exchange surface, activity of lipoprotein lipase, and FA binding protein levels $(15,16)$. Options that explain higher fetal LCPUFA contents are more-efficient trapping in the circulation or higher desaturation-chain elongation activity. Differences in placental blood flow and exchange surface may also affect transfer of other nutrients. Specific limitations of transplacental FA transfer or fetal FA handling may, however, cause low fetal EFA status only. This condition is considered a limiting factor in growth (17).

The present observation should be confirmed in a larger study, but it is in line with data from Koletzko and Braun (42). Studying premature infants of $33.6 \pm 1.4 \mathrm{wk}$, they found a positive relationship between birth weight and percentages of $20: 4 c, \omega 6$ and LCPUFA- $\omega 6$ in circulating fetal TG, whereas 18:3c, $\omega 3$ content was negatively related. Our results show that, among EFA, plasma CE $18: 3 c, \omega 3$ content is the only analyte that is higher in the infant with the lowest birth weight.

Acknowledgments. The authors thank Dr. F. P. L. van der Dijs for providing samples from the screening study on Curaçao, $\mathrm{H}$. J. R. Velvis for collecting data on birth weights, and M. Volmer for helping with the statistical analyses. We thank Prof. Dr. A. Okken, Dr. H. A. Woltil, Dr. H. A. Polman, and Dr. A. D. Siemensma for the disposal of fatty acid data from their study of human milk- and infant formula-fed newborns.

\section{REFERENCES}

1. Sprecher H 1981 Biochemistry of essential fatty acids. Prog Lipid Res 20:1322

2. Voss A, Reinhart M, Sankarappa S, Sprecher H 1991 The metabolism of 7,10,13,16,19-docosapentaenoic acid to 4,7,10,13,16,19-docosahexaenoic acid in rat liver is independent of a 4-desaturase. J Biol Chem 266:1999520000

3. Clandinin MT, Chappell JE, Leong S. Heim T, Swyer PR, Chance GW 1980 Intrauterine fatty acid accretion rates in human brain: implications for fatty acid requirements. Early Hum Dev 4:121-129

4. Clandinin MT, Chappell JE, Leong S, Heim T, Swyer PR, Chance GW 1980 Extrauterine fatty acid accretion in infant brain: implications for fatty acid requirements. Early Hum Dev 4:131-138

5. Martinez M 1992 Tissue levels of polyunsaturated fatty acids during early human development. J Pediatr 120(suppl):S129-S138

6. Farquharson J, Cockburn F, Patrick WA, Jamieson EC, Logan RW 1992 Infant cerebral cortex phospholipid fatty-acid composition and diet. Lancet 340:810-813

7. Clandinin MT, Chappell JE, Heim T, Swyer PR. Chance GW 1981 Fatty acid utilization in perinatal de novo synthesis of tissues. Early Hum Dev 5:355366

8. Clandinin MT, Chappell JE, Heim T 1982 Do low weight infants require nutrition with chain elongation-desaturation products of essential fatty acids? Prog Lipid Res 20:901-904

9. Innis SM 1991 Essential fatty acids in growth and development. Prog Lipid Res 30:39-103

10. Putnam JC, Carlson SE, DeVoe PW, Barness LA 1982 The effect of variations in dietary fatty acids on the fatty acid composition of erythrocyte phosphatidylcholine and phosphatidylethanolamine in human infants. Am J Clin Nutr 36:106-114

11. Clark KJ, Makrides M, Neumann MA, Gibson RA 1992 Determination of the optimal ratio of linoleic acid to $\alpha$-linolenic acid in infant formulas. J Pediatr 120(suppl):S151-S158

12. Koletzko B, Schmidt E, Bremer HJ, Haug M, Harzer G 1989 Effects of dietary long-chain polyunsaturated fatty acids on the essential fatty acid status of premature infants. Eur J Pediatr 148:669-675

13. Uauy RD, Birch DG. Birch EE, Tyson JE. Hoffman DR 1990 Effect of dietary omega-3 fatty acids on retinal function of very-low-birth-weight neonates. Pediatr Res 28:485-492

14. Lucas A, Morley R, Cole TJ, Lister G, Leeson-Payne C 1992 Breast milk and subsequent intelligence quotient in children born preterm. Lancet 339:261264

15. Coleman RA 1989 The role of the placenta in lipid metabolism and transport. Semin Perinatol 13:180-191

16. Kimura RE 1989 Fatty acid metabolism in the fetus. Semin Perinatol 13:202210

17. Feldman M, van Aerde JE, Clandinin MT 1992 Lipid accretion in the fetus and newborn. In: Polin RA, Fox WW (eds) Fetal and Neonatal Physiology. WB Saunders Co., Philadelphia, pp 299-314

18. Subbaiah PV, Monshizadegan H 1988 Substrate specificity of human plasma lecithin-cholesterol acyltransferase towards molecular species of phosphatidylcholine in native plasma. Biochim Biophys Acta 963:445-455

19. Christensen NC 1981 Free fatty acids, glycerol and triglycerides during the first 24 hours in infants with a birth weight $\leq 2700$ grams. Acta Paediatr Scand 70:485-490

20. Pikaar NA, Fernandes J 1966 Influence of different types of dietary fat on the fatty acid composition of some serum lipid fractions in infants and children. Am J Clin Nutr 19:194-204

21. Koletzko B, Müller J 1990 Cis- and trans-isomeric fatty acids in plasma lipids of newborn infants and their mothers. Biol Neonate 57:172-178

22. Friedman Z, Danon A, Lamberth EL, Mann WJ 1978 Cord blood fatty acid composition in infants and in their mothers during the third trimester. $J$ Pediatr 92:461-466

23. van Beusekom CM, Nijeboer HJ, van der Veere CN, Luteyn AJ, Sorhaindo B, Offringa PJ, Muskiet FAJ, Boersma ER 1993 Indicators of long chain polyunsaturated fatty acid status of exclusively breastfed infants at delivery and after 20-22 days. Early Hum Dev 32:207-218

24. van der Dijs FPL, van den Berg GA, Schermer JG, Muskiet FD, Landman H. Muskiet FAJ 1992 Screening cord blood for hemoglobinopathies and thalassemia by HPLC. Clin Chem 38:1864-1869

25. Hindriks FR, Wolthers BG, Groen A 1977 The determination of total cholesterol in serum by gas-liquid chromatography compared with two other methods. Clin Chim Acta 74:207-215

26. Folch J, Lees M, Sloane-Stanley GH 1957 A simple method for the isolation and purification of total lipids from animal tissues. J Biol Chem 226:497509

27. Hoving EB, Jansen G, Volmer M, van Doormaal JJ, Muskiet FAJ 1988 Profiling of plasma cholesterol ester and triglyceride fatty acids as their methyl esters by capillary gas chromatography, preceded by a rapid aminopropyl-silica column chromatographic separation of lipid classes. J Chromatogr 434:395-409

28. Muskiet FAJ, van Doormaal JJ, Martini IA. Wolthers BG, van der Slik W 1983 Capillary gas chromatographic profiling of total long-chain fatty acids and cholesterol in biological materials. J Chromatogr 278:231-244

29. Deming WE 1943 Statistical Adjustment of Data. John Wiley and Sons, New York, $p 184$

30. Lubchenco LO, Hansman C, Dressler M, Boyd E 1963 Intrauterine growth as estimated from liveborn birth-weight data at 24 to 40 weeks of gestation. Pediatrics 32:793-800

31. Hollingsworth DR, Cousins L 1982 Diabetes in pregnancy: a new perspective. 
In: Milunsky A, Friedman EA, Gluck L (eds) Advances in Perinatal Medicine. Vol 2. Plenum Medical Book Company, New York, pp 245-320

32. Jain SK 1985 Prematurity and lecithin-cholesterol acyltransferase deficiency in newborn infants. Pediatr Res 19:58-60

33. Amr S, Chowdhry P, Hamosh P, Hamosh M 1988 Low levels of apolipoprotein A1 are not contributors to the low lecithin-cholesterol acyl transferase activity in premature newborn infants. Pediatr Res 24:191-193

34. van Deenen LLM, de Gier J 1974 Lipids of the red cell membrane. In: Surgenor DMN (ed) The Red Blood Cell. 2nd Ed. Academic Press, New York, pp 147-211

35. Crawford MA, Hassam AG, Williams G, Whitehouse WL 1976 Essential fatty acids and fetal brain growth. Lancet 1:452-453

36. Laborda J, Naval J, Calvo M, Lampreave F, Uriel J 1989 Alpha-fetoprotein and albumin uptake by mouse tissues during development. Biol Neonate $56: 332-341$
37. Parmelee DC, Evenson MA, Deutsch HF 1978 The presence of fatty acids in human alpha-fetoprotein. J Biol Chem 253:2114-2119

38. Portman OW, Behrman RE, Soltys P 1969 Transfer of free fatty acids across the primate placenta. Am J Physiol 216:143-147

39. Holman RT, Johnson SB, Mercuri O, Itarte HJ, Rodrigo MA, De Thomas ME 1981 Essential fatty acid deficiency in malnourished children. Am J Clin Nutr 34:1534-1539

40. Al MDM, Hornstra G, van der Schouw YT, Bulstra-Ramakers MTEW, Huisjes HJ 1990 Biochemical EFA status of mothers and their neonates after normal pregnancy. Early Hum Dev 24:239-248

41. Lagarde M. Croset M. Sicard B. Dechavanne M 1986 Biological activities and metabolism of eicosenoic acids in relation to platelet and endothelial function. Prog Lipid Res 25:269-271

42. Koletzko B, Braun M 1991 Arachidonic acid and early human growth: is there a relation? Ann Nutr Metab 35:128-131 